\title{
Some Properties of $C R$-Submanifolds of a Nearly Trans-Sasakian Manifold with a Semi Symmetric Non-Metric Connection
}

\author{
Lovejoy S. Das', Mobin Ahmad'2, Abdul Haseeb² \\ ${ }^{1}$ Department of Mathematics, Kent State University, Kent, USA \\ ${ }^{2}$ Department of Mathematics, Integral University, Lucknow, India \\ Email: Idas@tusc.kent.edu, mobinahmad@rediffmail.com, malik haseeb@rediffmail.com
}

Received 30 May 2014; revised 30 June 2014; accepted 11 July 2014

Copyright (C) 2014 by authors and Scientific Research Publishing Inc.

This work is licensed under the Creative Commons Attribution International License (CC BY). http://creativecommons.org/licenses/by/4.0/

c) (7) Open Access

\begin{abstract}
This paper deals with the study of $C R$-submanifolds of a nearly trans-Sasakian manifold with a semi symmetric non-metric connection. Nijenhuis tensor, integrability conditions for some distributions on $C R$-submanifolds of a nearly trans-Sasakian manifold with a semi symmetric nonmetric connection are discussed.
\end{abstract}

\section{Keywords}

CR -Submanifolds, Nearly Trans-Sasakian Manifold, Semi Symmetric Non-Metric Connection, Distribution

\section{Introduction}

A. Bejancu defined and studied $C R$-submanifolds of a Kaehler manifold [1]. Later on, $C R$-submanifolds of a Sasakian manifold were studied by M. Kobayashi [2], K. Yano and M. Kon [3]. J. A. Oubina introduced a new class of almost contact metric manifold known as trans-Sasakian manifold [4]. This class contains $\alpha$-Sasakian and $\beta$-Kenmotsu manifold [5]. CR -submanifolds of a Kenmotsu manifold were studied by A. Bejancu and N. Papaghuic [6]. Geometry of $C R$-submanifolds of a trans-Sasakian manifold have been studied by M. H. Shahid in [7] [8]. CR -submanifolds of a nearly trans-Sasakian manifold were studied by Falleh R. Al-Solamy [9]. CR submanifolds of an LP-Sasakian manifold with a semi-symmetric metric connection were studied by M. Ahmad et al. [10]. Motivated by the studies in [11]-[13], in this paper we study $C R$-submanifolds of a nearly transSasakian manifold endowed with a semi symmetric non-metric connection.

Let $\nabla$ be a linear connection in an $n$-dimensional differentiable manifold $\bar{M}$. The torsion tensor $T$ of 
$\nabla$ is given by

$$
T(X, Y)=\nabla_{X} Y-\nabla_{Y} X-[X, Y] .
$$

The connection $\nabla$ is symmetric if torsion tensor $T$ vanishes, otherwise it is non-symmetric. The connection $\nabla$ is metric connection if there is a Riemannian metric $g$ in $M$ such that $\nabla g=0$, otherwise it is non-metric. It is well known that a linear connection is symmetric and metric if and only if it is the Levi-Civita connection.

In [14], S. Golab introduced the idea of a semi-symmetric and quarter symmetric linear connections. A linear connection $\nabla$ is said to be semi-symmetric if its torsion tensor $T$ is of the form

$$
T(X, Y)=\eta(Y) X-\eta(X) Y,
$$

where $\eta$ is a 1 -form and $\phi$ is a tensor field of the type $(1,1)$.

We consider integrabilities of horizontal and vertical distributions of $C R$-submanifolds with a semi symmetric non-metric connection. We also consider parallel horizontal distributions of $C R$-submanifolds.

The paper is organized as follows: In Section 2, we recall some necessary details of nearly trans-Sasakian manifold. In Section 3, we study $C R$-submanifolds of a nearly trans-Sasakian manifold. In Section 4, some useful lemmas are proved. In Section 5, some basic results on parallel distribution are investigated. In Section 6, we calculated Nijenhuis tensor and studied integrability conditions of the distributions on $C R$-submanifolds of a nearly trans-Sasakian manifold with a semi symmetric non-metric connection.

\section{Nearly Trans-Sasakian Manifold}

Let $\bar{M}$ be an $n$-dimensional almost contact metric manifold [15] with an almost contact metric structure $(\phi, \xi, \eta, g)$, that is, $\phi$ is a $(1,1)$ tensor field, $\xi$ is a vector field, $\eta$ is a 1 -form and $g$ is a compatible Riemannian metric such that

$$
\begin{gathered}
\phi^{2} X=-X+\eta(X) \xi, \quad \eta(\xi)=1, \phi \cdot \xi=0, \eta \cdot \phi=0 \\
g(\phi X, \phi Y)=g(X, Y)-\eta(X) \eta(Y) \\
g(X, \phi Y)=-g(\phi X, Y), \quad g(X, \xi)=\eta(X)
\end{gathered}
$$

for all vector fields $X, Y \in T \bar{M}$. There are two well known classes of almost contact metric manifolds, namely Sasakian and Kenmotsu manifolds. Sasakian manifolds are characterized by the tensorial relation

$$
\left(\overline{\bar{\nabla}}_{X} \phi\right) Y=g(X, Y) \xi-\eta(Y) X,
$$

while Kenmotsu manifolds are given by the tensor equation

$$
\left(\overline{\bar{\nabla}}_{X} \phi\right) Y=g(\phi X, Y) \xi-\eta(Y) \phi X .
$$

An almost contact metric structure $(\phi, \xi, \eta, g)$ on $\bar{M}$ is called a trans-Sasakian structure [4] if $(\bar{M} \times R, J, G)$ belongs to the class $W_{4}$ of Gray-Hervella classification of almost Hermitian manifolds [16], where $J$ is the almost complex structure on $\bar{M} \times R$ defined by

$$
J(X, a \mathrm{~d} / \mathrm{d} t)=(\phi X-a \xi, \eta(X) \mathrm{d} / \mathrm{d} t)
$$

for all vector fields $X$ on $M$ and smooth function $a$ on $\bar{M} \times R$. This may be expressed by the condition [17]

$$
\left(\overline{\bar{\nabla}}_{X} \phi\right) Y=\alpha[g(X, Y) \xi-\eta(Y) X]+\beta[g(\phi X, Y) \xi-\eta(Y) \phi X]
$$

for some smooth functions $\alpha$ and $\beta$ on $\bar{M}$ and we say that the trans-Sasakian structure is of type $(\alpha, \beta)$.

In 2000, C. Gherghe [18] introduced a nearly trans-Sasakian structure of the type $(\alpha, \beta)$ An almost contact metric structure $(\phi, \xi, \eta, g)$ on $\bar{M}$ is called a nearly trans-Sasakian structure [18] if

$$
\left(\overline{\bar{\nabla}}_{X} \phi\right) Y+\left(\overline{\bar{\nabla}}_{Y} \phi\right) X=\alpha[2 g(X, Y) \xi-\eta(Y) X-\eta(X) Y]-\beta[\eta(Y) \phi X+\eta(X) \phi Y] .
$$


A trans-Sasakian structure is always a nearly trans-Sasakian structure. Moreover, a nearly trans-Sasakian structure of type $(\alpha, \beta)$ is nearly Sasakian [19].

Let $M$ be an $m$-dimensional isometrically immersed submanifold of a nearly trans-Sasakian manifold $\bar{M}$ and denote by the same $g$ the Riemannian metric tensor field induced on $M$ from that of $\bar{M}$.

\section{CR -Submanifolds of Nearly Trans-Sasakian Manifolds}

Definition 3.1 An m-dimensional Riemannian submanifold $M$ of a nearly trans-Sasakian manifold $\bar{M}$ is called a CR-submanifold if $\xi$ is tangent to $M$ and there exists on $M$ a differentiable distribution $D: x \rightarrow D_{x} \subset T_{x}(M)$ such that

(i) the distribution $D_{x}$ is invariant under $\phi$, i.e., $\phi D_{x} \subset D_{x}$ for each $x \in M$;

(ii) The orthogonal complementary distribution $D^{\perp}: x \rightarrow D_{x}^{\perp} \subset T_{x}(M)$ of the distribution $D$ on $M$ is antiinvarient under $\phi$, i.e., $\phi D_{x}^{\perp}(M) \subset T_{x}^{\perp}(M)$ for all $x \in M$, where $T_{x}(M)$ and $T_{x}^{\perp}(M)$ are tangent space and normal space of $M$ at $x \in M$ respectively.

If $\operatorname{dim} D_{x}^{\perp}=0$ (resp., $\operatorname{dim} D_{x}=0$ ), then $C R$-submanifold is called an invariant (resp., anti-invariant). The distribution $D$ (resp., $D^{\perp}$ ) is called the horizontal (resp., vertical) distribution. The pair $\left(D, D^{\perp}\right)$ is called $\xi$-horizontal (resp., $\xi$-invariant) if $\xi_{x} \in D_{x}$ (resp., $\xi_{x} \in D_{x}^{\perp}$ ) for $x \in M$.

For any vector field $X$ tangent to $M$, we put

$$
X=P X+Q X,
$$

where $P X$ and $Q X$ belong to the distribution $D$ and $D^{\perp}$ respectively.

For any vector field $N$ normal to $M$, we put

$$
\phi N=B N+C N,
$$

where $B N$ (resp., $C N$ ) denotes the tangential (resp., normal) component of $\phi N$.

Now, we remark that owing to the existence of the 1 -form $\eta$, we can define a semi symmetric non-metric connection $\bar{\nabla}$ in any almost contact metric manifold by

$$
\bar{\nabla}_{X} Y=\overline{\bar{\nabla}}_{X} Y+\eta(Y) X
$$

such that $\left(\bar{\nabla}_{X} g\right)(Y, Z)=-\eta(Y) g(X, Z)-\eta(Z) g(X, Y)$ for any $X, Y \in T M$, where $\overline{\bar{\nabla}}$ is the induced connection with respect to $g$ on $M$.

By using (4) and (8), we get

$$
\left(\bar{\nabla}_{X} \phi\right) Y=\alpha[g(X, Y) \xi-\eta(Y) X]+\beta[g(\phi X, Y) \xi-\eta(Y) \phi X]-\eta(Y) \phi X .
$$

Similarly, we have

$$
\left(\bar{\nabla}_{Y} \phi\right) Y=\alpha[g(Y, X) \xi-\eta(X) Y]+\beta[g(\phi Y, X) \xi-\eta(X) \phi Y]-\eta(X) \phi Y .
$$

On adding above equations, we obtain

$$
\left(\bar{\nabla}_{X} \phi\right) Y+\left(\bar{\nabla}_{Y} \phi\right) X=\alpha[2 g(X, Y) \xi-\eta(X) Y-\eta(Y) X]-(\beta+1)[\eta(Y) \phi X-\eta(X) \phi Y] .
$$

This is the condition for $\bar{M}(\phi, \xi, \eta, g)$ with a semi symmetric non-metric connection to be nearly transSasakian manifold.

We denote by $g$ the metric tensor of $\bar{M}$ as well as that induced on $M$. Let $\bar{\nabla}$ be the semi symmetric non-metric connection on $\bar{M}$ and $\nabla$ be the induced connection on $M$ with respect to the unit normal $N$. Then we have:

Theorem 3.2 (i) If $M$ is $\xi$-horizontal, $X, Y \in D$ and $D$ is parallel with respect to $\nabla$, then the connection induced on a $C R$-submanifold of a nearly trans-Sasakian manifold with a semi symmetric non-metric connection is also a semi symmetric non-metric connection.

(ii) If $M$ is $\xi$-vertical, $X, Y \in D^{\perp}$ and $D^{\perp}$ is parallel with respect to $\nabla$, then the connection induced on a $C R$-submanifold of a nearly trans-Sasakian with a semi symmetric non-metric connection is also a semi symmetric non-metric connection.

(iii) The Gauss formula with respect to the semi symmetric non-metric connection is of the form 


$$
\bar{\nabla}_{X} Y=\nabla_{X} Y+h(X, Y) .
$$

Proof. Let $\nabla$ be the induced connection with respect to the unit normal $N$ on a $C R$-submanifold of a nearly trans-Sasakian manifold from a semi symmetric non-metric connection connection $\bar{\nabla}$, then

$$
\bar{\nabla}_{X} Y=\nabla_{X} Y+m(X, Y),
$$

where $m$ is a tensor field of the type $(0,2)$ on $C R$-submanifold $M$. If $\nabla^{\mathfrak{a}}$ be the induced connection on $C R$-submanifold from Riemannian connection $\overline{\bar{\nabla}}$, then

$$
\overline{\bar{\nabla}}_{X} Y=\nabla_{X}^{a ̊ a} Y+h(X, Y),
$$

where $h$ is a second fundamental form. By the definition of the semi symmetric non-metric connection, we have

$$
\bar{\nabla}_{X} Y=\overline{\bar{\nabla}}_{X} Y+\eta(Y) X .
$$

Now, using (11) and (12) in above equation, we have

$$
\nabla_{X} Y+m(X, Y)=\nabla_{X}^{\text {å }} Y+h(X, Y)+\eta(Y) X .
$$

Using (6), the above equation can be written as

$$
P \nabla_{X} Y+Q \nabla_{X} Y+m(X, Y)=P \nabla_{X}^{a ̊} Y+Q \nabla_{X}^{a ̊} Y+h(X, Y)+\eta(Y) P X+\eta(Y) Q X .
$$

From (13), comparing the tangential and normal components from both the sides, we get

$$
\begin{gathered}
h(X, Y)=m(X, Y) \\
P \nabla_{X} Y-\eta(Y) P X=P \nabla_{X}^{a ̊} Y \\
Q \nabla_{X} Y-\eta(Y) Q X=Q \nabla_{X}^{a} Y .
\end{gathered}
$$

Using (14), the Gauss formula for a $C R$-submanifold of a nearly trans-Sasakian manifold with a semi symmetric non-metric connection is

$$
\bar{\nabla}_{X} Y=\nabla_{X} Y+h(X, Y) .
$$

This proves (iii). In view of (15), if $M$ is $\xi$-horizontal, $X, Y \in D$ and $D$ is parallel with respect to $\nabla$, then the connection induced on a $C R$-submanifold of a nearly trans-Sasakian manifold with a semi symmetric non-metric connection is also a semi symmetric non-metric connection.

Similarly, using (16), if $M$ is $\xi$-vertical, $X, Y \in D^{\perp}$ and $D^{\perp}$ is parallel withrespect to $\nabla$, then the connection induced on a $C R$-submanifold of a nearly trans-Sasakian manifold with a semi symmetric nonmetric connection is also a semi symmetric non-metric connection.

Weingarten formula is given by

$$
\bar{\nabla}_{X} N=-A_{N} X+\nabla_{X}^{\perp} N+\eta(N) X
$$

for $X, Y \in T M, N \in T^{\perp} M, h: T M \times T M \rightarrow T M^{\perp}$ (resp., $A_{N}: T M \rightarrow T M$ ) is the second fundamental form (resp., tensor) of $M$ in $\bar{M}$ and $\nabla^{\perp}$ denotes the operator of the normal connection. Moreover, we have

$$
g(h(X, Y), N)=g\left(A_{N} X, Y\right) \text {. }
$$

\section{Some Basic Lemmas}

Lemma 4.1 Let $M$ be a $C R$-submanifold of a nearly trans-Sasakian manifold $\bar{M}$ with a semi symmetric non-metric connection. Then

$$
\begin{aligned}
& P\left(\nabla_{X} \phi P Y\right)+P\left(\nabla_{Y} \phi P X\right)-P\left(A_{\phi Q Y} X\right)-P\left(A \phi_{\varphi Q X} Y\right) \\
& \quad=\phi P \nabla_{X} Y+\phi P \nabla_{Y} X+2 \alpha g(X, Y) P \xi-\alpha \eta(X) P Y-\alpha \eta(Y) P X-(\beta+1)[\eta(Y) \phi P X+\eta(X) \phi P Y]
\end{aligned}
$$




$$
\begin{gathered}
Q\left(\nabla_{X} \phi P Y\right)+Q\left(\nabla_{Y} \phi P X\right)-Q\left(A_{\phi Q Y} X\right)-Q\left(A \phi_{\varphi Q X} Y\right) \\
=2 \alpha g(X, Y) Q \xi-\alpha \eta(X) Q Y-\alpha \eta(Y) Q X+2 B h(X, Y) \\
h(X, \phi P Y)+h(Y, \phi P X)+\nabla_{X}^{\perp} \phi Q Y+\nabla_{Y}^{\perp} \phi Q X \\
=\phi\left(Q \nabla_{X} Y\right)+\phi\left(Q \nabla_{Y} X\right)+2 C h(X, Y)-(\beta+1)[\eta(Y) \phi Q X+\eta(X) \phi Q Y]
\end{gathered}
$$

for $X, Y \in T M$.

Proof. By direct covariant differentiation, we have

$$
\bar{\nabla}_{X} \phi Y=\left(\bar{\nabla}_{X} \phi\right) Y+\phi\left(\bar{\nabla}_{X} Y\right) \text {. }
$$

By virtue of (6), (9), (17) and (18), we get

$$
\begin{aligned}
\nabla_{X} \phi P Y+h(X, \phi P Y)-A_{\phi Q Y} X+\nabla_{X}^{\perp} \phi Q Y-\phi[V]= & \alpha[g(X, Y) \xi-\eta(Y) X] \\
& +\beta g(\phi X, Y) \xi-(\beta+1) \eta(Y) \phi X .
\end{aligned}
$$

Similarly, we have

$$
\begin{aligned}
\nabla_{Y} \phi P X+h(Y, \phi P X)-A_{\phi Q X} Y+\nabla_{Y}^{\perp} \phi Q X-\phi\left[\nabla_{Y} X+h(X, Y)\right]= & \alpha[g(X, Y) \xi-\eta(X) Y] \\
& +\beta g(\phi Y, X) \xi-(\beta+1) \eta(X) \phi Y .
\end{aligned}
$$

On adding above equations, we have

$$
\begin{gathered}
\nabla_{X} \phi P Y+\nabla_{Y} \phi P X+h(X, \phi P Y)+h(Y, \phi P X)-A_{\phi Q Y} X-A_{\phi Q X} Y+\nabla_{X}^{\perp} \phi Q Y+\nabla_{Y}^{\perp} \phi Q X-\phi \nabla_{X} Y-\phi \nabla_{Y} X \\
-2 \phi h(X, Y)=\alpha[2 g(X, Y) \xi-\eta(v) Y-\eta(Y) X]-(\beta+1)[\eta(X) \phi Y+\eta(Y) \phi X] .
\end{gathered}
$$

Now using (6), (7) and equating horizontal, vertical and normal components in above equation, the lemma follows.

Lemma 4.2 Let $M$ be a CR-submanifold of a nearly trans-Sasakian manifold $\bar{M}$ with a semi symmetric non-metric connection. Then

$$
\begin{aligned}
2\left(\bar{\nabla}_{X} \phi\right) Y= & \nabla_{X} \phi Y-\nabla_{Y} \phi X+h(X, \phi Y)-h(Y, \phi X)-\phi[X, Y]+\alpha[2 g(X, Y) \xi-\eta(Y) X-\eta(X) Y] \\
& -(\beta+1)[\eta(Y) \phi X+\eta(X) \phi Y]
\end{aligned}
$$

for any $X, Y \in D$.

Proof. By the use of (17), we have

$$
\bar{\nabla}_{X} \phi Y-\bar{\nabla}_{Y} \phi X=\nabla_{X} \phi Y-\nabla_{Y} \phi X+h(X, \phi Y)-h(Y, \phi X) .
$$

Also, we have

$$
\bar{\nabla}_{X} \phi Y-\bar{\nabla}_{Y} \phi X=\left(\bar{\nabla}_{X} \phi\right) Y-\left(\bar{\nabla}_{Y} \phi\right) X+\phi[X, Y] .
$$

From above equations, we get

$$
\left(\bar{\nabla}_{X} \phi\right) Y-\left(\bar{\nabla}_{Y} \phi\right) X=\nabla_{X} \phi Y-\nabla_{Y} \phi X+h(X, \phi Y)-h(Y, \phi X)-\phi[X, Y] .
$$

For a nearly trans-Sasakian manifold with a semi symmetric non-metric connection, we have

$$
\left(\bar{\nabla}_{X} \phi\right) Y+\left(\bar{\nabla}_{Y} \phi\right) X=\alpha[2 g(X, Y) \xi-\eta(X) Y-\eta(Y) X]-(\beta+1)[\eta(Y) \phi X+\eta(X) \phi Y] .
$$

Combining (26) and (27), the lemma follows.

In particular, we have the following corollary.

Corollary 4.3 Let $M$ be a $\xi$-vertical $C R$-submanifold of a nearly trans-Sasakian manifold $\bar{M}$ with a semi symmetric non-metric connection. Then

$$
2\left(\bar{\nabla}_{X} \phi\right) Y=\nabla_{X} \phi Y-\nabla_{Y} \phi X+h(X, \phi Y)-h(Y, \phi X)-\phi[X, Y]+2 \alpha g(X, Y) \xi
$$

for any $X, Y \in D$. 
Similarly, by Weingarten formula, we can easily get the following lemma.

Lemma 4.4 Let $M$ be a CR -submanifold of a nearly trans-Sasakian manifold $\bar{M}$ with a semi symmetric non-metric connection. Then

$$
\begin{aligned}
2\left(\bar{\nabla}_{Y} \phi\right) Z= & A_{\phi Y} Z-A_{\phi Z} Y+\nabla_{Y}^{\perp} \phi Z-\nabla_{Z}^{\perp} \phi Y-\phi[Y, Z]+\alpha[2 g(X, Y) \xi-\eta(Y) Z-\eta(Z) Y] \\
& -(\beta+1)[\eta(Z) \phi Y+\eta(Y) \phi Z]
\end{aligned}
$$

for any $Y, Z \in D$.

Corollary 4.5 Let $M$ be a $\xi$-horizontal $C R$-submanifold of a nearly trans-Sasakian manifold $\bar{M}$ with a semi symmetric non-metric connection. Then

$$
2\left(\bar{\nabla}_{Y} \phi\right) Z=A_{\phi Y} Z-A_{\phi Z} Y+\nabla_{Y}^{\perp} \phi Z-\nabla_{Z}^{\perp} \phi Y-\phi[Y, Z]+2 \alpha g(X, Y) \xi
$$

for any $Y, Z \in D^{\perp}$.

Lemma 4.6 Let $M$ be a CR-submanifold of a nearly trans-Sasakian manifold $\bar{M}$ with a semi symmetric non-metric connection. Then

$$
\begin{aligned}
2\left(\bar{\nabla}_{X} \phi\right) Y= & -A_{\phi Y} X+\nabla_{X}^{\perp} \phi Y-h(Y, \phi X)-\nabla_{Y} \phi X-\phi[X, Y]+\alpha[2 g(X, Y) \xi-\eta(Y) X-\eta(X) Y] \\
& -(\beta+1)[\eta(Y) \phi X+\eta(X) \phi Y]
\end{aligned}
$$

for any $X \in D, Y \in D^{\perp}$.

Proof. As we have

$$
\left(\bar{\nabla}_{X} \phi\right) Y=\nabla_{X} \phi Y-\phi\left(\bar{\nabla}_{X} Y\right) .
$$

Now, by using Gauss and Weingarten formulae in above equation, we have

$$
\left(\bar{\nabla}_{X} \phi\right) Y=-A_{\phi Y} X+\nabla_{X}^{\perp} \phi Y-\phi \nabla_{X} Y-\phi h(X, Y) .
$$

Also, we have

$$
\left(\bar{\nabla}_{Y} \varphi\right) X=\nabla_{Y} \phi X+h(Y, \phi X)-\phi \nabla_{Y} Y-\phi h(X, Y) .
$$

From above equations, we get

$$
\left(\bar{\nabla}_{X} \phi\right) Y-\bar{\nabla}_{Y} \phi X=-A_{\phi Y} X+\nabla_{X}^{\perp} \phi Y-\phi[X, Y]-\nabla_{Y} \phi X-h(Y, \phi X) .
$$

In view of (10) and above equation, the lemma follows.

\section{Parallel Distributions}

Definition 5.1 The horizontal (resp., vertical) distribution $D$ (resp., $D^{\perp}$ ) is said to be parallel [1] with respect to the semi symmetric non-metric connection $\nabla$ on $M$ if $\nabla_{X} Y \in D$ (resp., $\nabla_{Z} W \in D^{\perp}$ ) for any $X, Y \in D$ (resp., $W, Z \in D^{\perp}$ ).

Now, we have the following proposition.

Proposition 5.2 Let $M$ be a $\xi$-vertical $C R$-submanifold of a nearly trans-Sasakian manifold $\bar{M}$ with a semi symmetric non-metric connection. Then

$$
h(X, \phi Y)=h(Y, \phi X)
$$

for all $X, Y \in D$.

Proof. By the parallelness of horizontal distribution $D$, we have

$$
\nabla_{X} \phi Y \in D, \nabla_{Y} \phi X \in D \text { for any } X, Y \in D .
$$

For $Y \in D$, using the fact that $Q X=Q Y=0$, (21) gives

$$
B h(X, Y)=g(X, Y) Q \xi \text { for any } X, Y \in D .
$$

Therefore in view of (7), we have

$$
\phi h(X, Y)=g(X, Y) Q \xi+C h(X, Y) \text { for any } X, Y \in D .
$$


From (22), we have

$$
h(X, \phi Y)+h(Y, \phi X)=2 \phi h(X, Y)-2 g(X, Y) Q \xi
$$

for any $X, Y \in D$.

Now, putting $X=\phi X \in D$ and $Y=\phi Y \in D$ in (36), we get respectively

$$
\begin{aligned}
& h(\phi X, \phi Y)-h(Y, X)=2 \phi h(\phi X, Y)-2 g(\phi X, Y) Q \xi \\
& h(\phi X, \phi Y)-h(Y, X)=2 \phi h(X, \phi Y)-2 g(X, \phi Y) Q \xi
\end{aligned}
$$

Hence from (37) and (38), we have

$$
\phi h(X, \phi Y)-\phi h(Y, \phi X)=g(X, \phi Y) Q \xi-g(\phi X, Y) Q \xi .
$$

Operating $\phi$ on both sides of (39) and using $\phi \xi=0$, we get

$$
h(X, \phi Y)=h(Y, \phi X)
$$

for all $X, Y \in D$.

Now, for the distribution $D^{\perp}$, we have the following proposition.

Proposition 5.3 Let $M$ be a $\xi$-vertical CR-submanifold of a nearly trans-Sasakian manifold $\bar{M}$ with a semi symmetric non-metric connection. If the distribution $D^{\perp}$ is parallel with a semi symmetric non-metric connection on $M$. Then

$$
\left(A_{\varphi Y} Z+A_{\varphi Z} Y\right) \in D^{\perp} \text { for any } Y, Z \in D^{\perp} .
$$

Proof. By using Weingarten formula, we have

$$
\bar{\nabla}_{Y} \phi Z=-A_{\phi Z} Y+\nabla_{Y}^{\perp} \phi Z
$$

and

$$
\bar{\nabla}_{Z} \phi Y=-A_{\phi Y} Z+\nabla_{Z}^{\perp} \phi Y
$$

for $Y, Z \in D^{\perp}$. From above equations, we have

$$
-A_{\phi Z} Y+\nabla_{Y}^{\perp} \phi Z-A_{\phi Y} Z+\nabla_{Z}^{\perp} \phi Y=\left(\bar{\nabla}_{Y} \phi\right) Z+\left(\bar{\nabla}_{Z} \phi\right) Y+\phi\left(\bar{\nabla}_{Y} Z+\bar{\nabla}_{Z} Y\right) \text {. }
$$

Using (10) and (17), we obtain

$$
\begin{aligned}
-A_{\phi Z} Y+\nabla_{Y}^{\perp} \phi Z-A_{\phi Y} Z+\nabla_{Z}^{\perp} \phi Y= & \alpha[2 g(Y, Z) \xi-\eta(Y) Z-\eta(Z) Y]-(\beta+1)[\eta(Y) \phi Z+\eta(Z) \phi Y] \\
& +\phi \nabla_{Y} Z+\phi \nabla_{Z} Y+2 \phi h(Y, Z)
\end{aligned}
$$

for any $Y, Z \in D^{\perp}$. Taking inner product with $X \in D$ in (41), we get

$$
g\left(A_{\phi Z} Y, X\right)+g\left(A_{\phi Y} Z, X\right)=g\left(\nabla_{Y} Z, \phi X\right)+g\left(\nabla_{Z} Y, \phi X\right) .
$$

If the distribution $D^{\perp}$ is parallel, then $\nabla_{Y} Z \in D^{\perp}$ and $\nabla_{Z} Y \in D^{\perp}$ for any $Y, Z \in D^{\perp}$. So from above equation, we get

$$
g\left(A_{\phi Z} Y, X\right)+g\left(A_{\phi Y} Z, X\right)=0
$$

or

$$
g\left(A_{\phi Z} Y+A_{\phi Y} Z, X\right)=0
$$

which implies that $A_{\varphi Z} Y+A_{\varphi Y} Z \in D^{\perp}$ for any $Y, Z \in D^{\perp}$.

Definition 5.4 A CR -submanifold with a semi symmetric non-metric connection is said to be mixed totally geodesic if $h(X, Z)=0$ for all $X \in D$ and $Z \in D^{\perp}$.

Definition 5.5 A normal vector field $N \neq 0$ with a semi symmetric non-metric connection is called $D$ -parallel normal section if $\nabla_{X}^{\perp} N=0$ for all $X \in D$.

Now, we have the following proposition. 
Proposition 5.6 Let $M$ be a mixed totally geodesic $\xi$-vertical $C R$-submanifold of a nearly transSasakian manifold $\bar{M}$ with a semi symmetric non-metric connection. Then the normal section $N \in \phi D^{\perp}$ is $D$-parallel if and only if $\nabla_{X} \phi N \in D$ for all $X \in D$.

\section{Integrability Conditions of Distributions}

In this section, we calculate the Nijenhuis tensor $N(X, Y)$ on a nearly trans-Sasakian manifold $\bar{M}$ with a semi symmetric non-metric connection. For this, first we prove the following lemma.

Lemma 6.1 Let $\bar{M}$ be a nearly trans-Sasakian manifold with a semi symmetric non-metric connection. Then

$$
\begin{aligned}
\left(\bar{\nabla}_{\phi X} \phi\right) Y= & 2 \alpha g(\phi X, Y) \xi-\alpha \eta(Y) \phi X+(\beta+1) \eta(Y) X-(\beta+1) \eta(X) \eta(Y) \xi-\eta(X) \bar{\nabla}_{Y} \xi \\
& +\phi\left(\bar{\nabla}_{Y} \phi\right) X-\left(\left(\bar{\nabla}_{Y} \eta\right) X\right) \xi
\end{aligned}
$$

for any $X, Y \in T \bar{M}$.

Proof. From the definition of nearly trans-Sasakian manifold with a semi symmetric non-metric connection $\bar{M}$, we have

$$
\left(\bar{\nabla}_{\phi X} \phi\right) Y=2 \alpha g(\phi X, Y) \xi-\alpha \eta(Y) \phi X+(\beta+1) \eta(Y) X-(\beta+1) \eta(X) \eta(Y) \xi-\left(\bar{\nabla}_{Y} \phi\right) \phi X .
$$

Also, we have

$$
\left(\bar{\nabla}_{Y} \phi\right) \phi X=\eta(X) \bar{\nabla}_{Y} \xi-\phi\left(\bar{\nabla}_{Y} \phi\right) X+\left(\left(\bar{\nabla}_{Y} \eta\right) X\right) \xi .
$$

Now, using (48) in (47), we get

$$
\begin{aligned}
\left(\bar{\nabla}_{\phi X} \phi\right) Y= & 2 \alpha g(\phi X, Y) \xi-\alpha \eta(Y) \phi X+(\beta+1) \eta(Y) X-(\beta+1) \eta(X) \eta(Y) \xi-\eta(X) \bar{\nabla}_{Y} \xi \\
& +\phi\left(\bar{\nabla}_{Y} \phi\right) X-\left(\left(\bar{\nabla}_{Y} \eta\right) X\right) \xi
\end{aligned}
$$

for any $X, Y \in T \bar{M}$, which completes the proof of the lemma.

On a nearly trans-Sasakian manifold with a semi symmetric non-metric connection $\bar{M}$, Nijenhuis tensor is given by

$$
N(X, Y)=\left(\bar{\nabla}_{\phi X} \phi\right) Y-\left(\bar{\nabla}_{\phi Y} \phi\right) X-\phi\left(\bar{\nabla}_{X} \phi\right) Y+\phi\left(\bar{\nabla}_{Y} \phi\right) X
$$

for any $X, Y \in T \bar{M}$.

From (46) and (50), we get

$$
\begin{aligned}
N(X, Y)= & 4 \alpha g(\phi X, Y) \xi-\alpha[\eta(Y) \phi X-\eta(X) \phi Y]+(\beta+1)[\eta(Y) X-\eta(X) Y] \\
& -\eta(X) \bar{\nabla}_{Y} \xi+\eta(Y) \bar{\nabla}_{X} \xi+2 g(X, \phi Y) \xi+2 \phi\left(\bar{\nabla}_{Y} \phi\right) X-2 \phi\left(\bar{\nabla}_{X} \phi\right) Y .
\end{aligned}
$$

In view of (10), we have

$$
\varphi\left(\bar{\nabla}_{X} \phi\right) Y=-\alpha \eta(Y) \phi X-\alpha \eta(X) \phi Y+(\beta+1)[\eta(Y) X+\eta(X) Y]-2(\beta+1) \eta(X) \eta(Y) \xi-\phi\left(\bar{\nabla}_{Y} \phi\right) X .
$$

Using above equation in (51), we obtain

$$
\begin{aligned}
N(X, Y)= & 4 \alpha g(\phi X, Y) \xi+\alpha \eta(Y) \phi X+3 \alpha \eta(X) \phi Y-(\beta+1) \eta(Y) X-3(\beta+1) \eta(X) Y \\
& +2 g(X, \phi Y) \xi+4 \phi\left(\bar{\nabla}_{Y} \phi\right) X+4(\beta+1) \eta(X) \eta(Y) \xi-\eta(X) \bar{\nabla}_{Y} \xi+\eta(Y) \bar{\nabla}_{X} \xi
\end{aligned}
$$

for any $X, Y \in T \bar{M}$.

Proposition 6.2 Let $M$ be a $\xi$-vertical $C R$-submanifold of a nearly trans-Sasakian manifold $\bar{M}$ with a semi symmetric non-metric connection. Then the distribution $D$ is integrable if the following conditions are satisfied:

$$
S(X, Z) \in D, h(X, Z)=h(\phi X, Z)
$$

for any $X, Z \in D$.

Proof. The torsion tensor $S(X, Y)$ of the almost contact metric structure $(\phi, \xi, \eta, g)$ is given by 


$$
S(X, Y)=N(X, Y)+2 \mathrm{~d} \eta(X, Y) \xi=N(X, Y)+2 g(\phi X, Y) \xi .
$$

Thus, we have

$$
S(X, Y)=[\phi X, \phi Y]-\phi[\phi X, Y]-\phi[X, \phi Y]+2 g(\phi X, Y) \xi
$$

for any $X, Y \in T M$.

Suppose that the distribution $D$ is integrable. So for $X, Y \in D, Q[X, Y]=0$. If $S(X, Y) \in D$, then from (52) and (54), we have

$$
4 \alpha g(\phi X, Y) Q \xi+4\left(\phi Q \nabla_{Y} \phi X+\phi h(Y, \phi X)+Q \nabla_{Y} X+h(X, Y)\right)=0
$$

for any $X, Y \in D$ and $\xi \in D^{\perp}$.

Replacing $Y$ by $\phi Z$ for $Z \in D$, we get

$$
4 \alpha g(\phi X, \phi Z) Q \xi+4\left(\phi Q \nabla_{\phi Z} \varphi X+\phi h(\phi Z, \phi X)+Q \nabla_{\phi Z} X+h(X, \phi Z)\right)=0 .
$$

Interchanging $X$ and $Z$ for $X, Z \in D$ in (57), we have

$$
4 \alpha g(\phi Z, \phi X) Q \xi+4\left(\phi Q \nabla_{\phi X} \phi Z+\phi h(\phi X, \phi Z)+Q \nabla_{\phi X} Z+h(Z, \phi X)\right)=0 .
$$

Subtracting above equations, we get

$$
\phi Q[\phi X, \phi Z]+Q[X, \phi Z]+h(X, \phi Z)-h(Z, \phi X)=0
$$

for any $X, Z \in D$ and the assertion follows.

Now, we prove the following proposition.

Proposition 6.3 Let $M$ be a CR -submanifold of a nearly trans-Sasakian manifold $\bar{M}$ with a semi symmetric non-metric connection. Then

$$
A_{\phi Y} Z-A_{\phi Z} Y=\frac{1}{3} \phi P[Y, Z]+\alpha(\eta(Y) Z-\eta(Z) Y)
$$

for any $Y, Z \in D^{\perp}$.

Proof. For $Y, Z \in D^{\perp}$ and $X \in T M$, we have

$$
\begin{aligned}
2 g\left(A_{\phi Z} Y, X\right)= & 2 g(h(X, Y), \phi Z) \\
2 g\left(A_{\phi Z} Y, X\right)= & g(h(X, Y), \phi Z)+g(h(X, Y), \phi Z) \\
2 g\left(A_{\phi Z} Y, X\right)= & g\left(\bar{\nabla}_{X} Y+\bar{\nabla}_{Y} X, \phi Z\right) \\
2 g\left(A_{\phi Z} Y, X\right)= & -g\left(\phi\left(\bar{\nabla}_{X} Y+\bar{\nabla}_{Y} X\right), Z\right) \\
2 g\left(A_{\phi Z} Y, X\right)= & -g\left[\left(\bar{\nabla}_{Y} \phi X+\bar{\nabla}_{X} \phi Y\right)-\alpha(2 g(X, Y) \xi-\eta(X) Y-\eta(Y) X)\right. \\
& +(\beta+1)(\eta(Y) \phi X+\eta(X) \phi Y), Z] \\
2 g\left(A_{\phi Z} Y, X\right)= & -g\left(\bar{\nabla}_{Y} \phi X, Z\right)-g\left(\bar{\nabla}_{X} \phi Y, Z\right)+2 \alpha g(X, Y) \eta(Z)-\alpha g(X, Z) \eta(Y)-\alpha g(Y, Z) \eta(X) \\
2 g\left(A_{\phi Z} Y, X\right)= & g\left(\bar{\nabla}_{Y} Z, \phi X\right)+g\left(A_{\phi Y} Z, X\right)+2 \alpha g(X, Y) \eta(Z)-\alpha g(X, Z) \eta(Y)-\alpha g(Y, Z) \eta(X) .
\end{aligned}
$$

The above equation is true for all $X \in T M$, therefore transvecting the vector field $X$ both sides, we obtain

$$
2 A_{\phi Z} Y=A_{\phi Y} Z-\phi \bar{\nabla}_{Y} Z-\alpha g(Y, Z) \xi-\alpha \eta(Y) Z+2 \alpha \eta(Z) Y .
$$

Interchanging the vector fields $Y$ and $Z$, we get

$$
2 A_{\phi Y} Z=A_{\phi Z} Y-\phi \bar{\nabla}_{Z} Y-\alpha g(Y, Z) \xi-\alpha \eta(Z) Y+2 \alpha \eta(Y) Z .
$$

From (62) and (63), we get

$$
A_{\phi Y} Z-A_{\phi Z} Y=\frac{1}{3} \phi P[Y, Z]+\alpha(\eta(Y) Z-\eta(Z) Y)
$$

for any $Y, Z \in D^{\perp}$, which completes the proof. 
Proposition 6.4 Let $M$ be a CR-submanifold of a nearly trans-Sasakian manifold $\bar{M}$ with a semi symmetric non-metric connection. Then the distribution $D^{\perp}$ is integrable if and only if

$$
A_{\phi Y} Z-A_{\phi Z} Y=\alpha(\eta(Y) Z-\eta(Z) Y)
$$

for $Y, Z \in D^{\perp}$.

Proof. Proof of the theorem is similar as proof of the theorem 5.4 of [2].

Corollary 6.5 Let $M$ be a $\xi$-horizontal CR-submanifold of a nearly trans-Sasakian manifold $\bar{M}$ with a semi symmetric non-metric connection. Then the distribution $D^{\perp}$ is integrable if and only if

$$
A_{\phi Y} Z-A_{\phi Z} Y=0
$$

for $Y, Z \in D^{\perp}$.

\section{References}

[1] Bejancu, A. (1978) CR -Submanifolds of a Kaehler Manifold I. Proceedings of the American Mathematical Society, 69, $135-142$.

[2] Kobayashi, M. (1981) CR-Submanifolds of Sasakian Manifold. Tensor, N. S., 35, 297-307.

[3] Yano, K. and Kon, M. (1989) On Contact CR-Submanifolds. Journal of the Korean Mathematical Society, 26, 231262.

[4] Oubina, J.A. (1985) New Class of Almost Contact Metric Structures. Publicationes Mathematicae Debrecen, 32, 187193.

[5] Janssens, D. and Vanhecke, L. (1981) Almost Contact Structures and Curvature Tensors. Kodai Mathematical Journal, 1, 1-27. http://dx.doi.org/10.2996/kmj/1138036310

[6] Bejancu, A. and Papaghuic, N. (1984) CR -Submanifolds of a Kenmotsu Manifold. Rendiconti di Matematica, 7, 607622.

[7] Shahid, M.H. (1991) CR-Submanifolds of a Trans-Sasakian Manifold. Indian Journal of Pure and Applied Mathematics, 22, 1007-1012.

[8] Shahid, M.H. (1994) CR-Submanifolds of a Trans-Sasakian Manifold II. Indian Journal of Pure and Applied Mathematics, 25, 299-307.

[9] Al-Solamy, F.R. (2002) CR -Submanifolds of a Nearly Trans-Sasakian Manifold. IJMMS, 31, 167-175.

[10] Ahmad, M., Özgür, C. and Haseeb, A. CR -Submanifolds of an LP-Sasakian Manifold with a Semi-Symmetric Metric Connection. Hacettepe Journal of Mathematics and Statistics.

[11] Das, L.S. and Ahmad, M. (2009) CR -Submanifolds of an LP -Sasakian Manifold with a Quarter Symmetric NonMetric Connection. Mathematical Sciences Research Journal, 13, 161-169.

[12] Das, L.S., Ahmad, M. and Haseeb, A. (2011) Semi-Invariant Submanifolds of a Nearly Sasakian Manifold Endowed with a Semi-Symmetric Non-Metric Connection. Journal of Applied Analysis, 17, 1-12.

[13] Tripathi, M.M. (2004) On Semi-Invariant Submanifolds of Nearly Trans-Sasakian Manifolds. The International Journal of Pure and Applied Mathematical Sciences, 1, 15-34.

[14] Golab, S. (1975) On Semi-Symmetric and Quarter Symmetric Linear Connections. Tensor, N. S., 29, $249-254$.

[15] Blair, D.E. (1976) Contact Manifolds in Riemannian Geometry, Lecture Notes in Mathematics. Vol. 509, SpringerVerlag, Berlin.

[16] Gray, A. and Hervella, L.M. (1980) The Sixteen Classes of Almost Hermitian Manifolds and Their Linear Invariants. Annali di Matematica Pura ed Applicata, 4, 35-58. http://dx.doi.org/10.1007/BF01796539

[17] Blair, D.E. and Oubina, J.A. (1990) Conformal and Related Changes of Metric on the Product of Two Almost Contact Metric Manifolds. Publications Mathematiques, 34, 199-207. http://dx.doi.org/10.5565/PUBLMAT_34190_15

[18] Gherghe, C. (2000) Harmonicity on Nearly Trans-Sasaki Manifolds. Demonstratio Mathematica, 33, 151-157.

[19] Blair, D.E., Showers, D.K. and Yano, K. (1996) Nearly Sasakian Structures. Kodai Mathematical Journal, 27, 175180. 
Scientific Research Publishing (SCIRP) is one of the largest Open Access journal publishers. It is currently publishing more than 200 open access, online, peer-reviewed journals covering a wide range of academic disciplines. SCIRP serves the worldwide academic communities and contributes to the progress and application of science with its publication.

Other selected journals from SCIRP are listed as below. Submit your manuscript to us via either submit@scirp.org or Online Submission Portal.
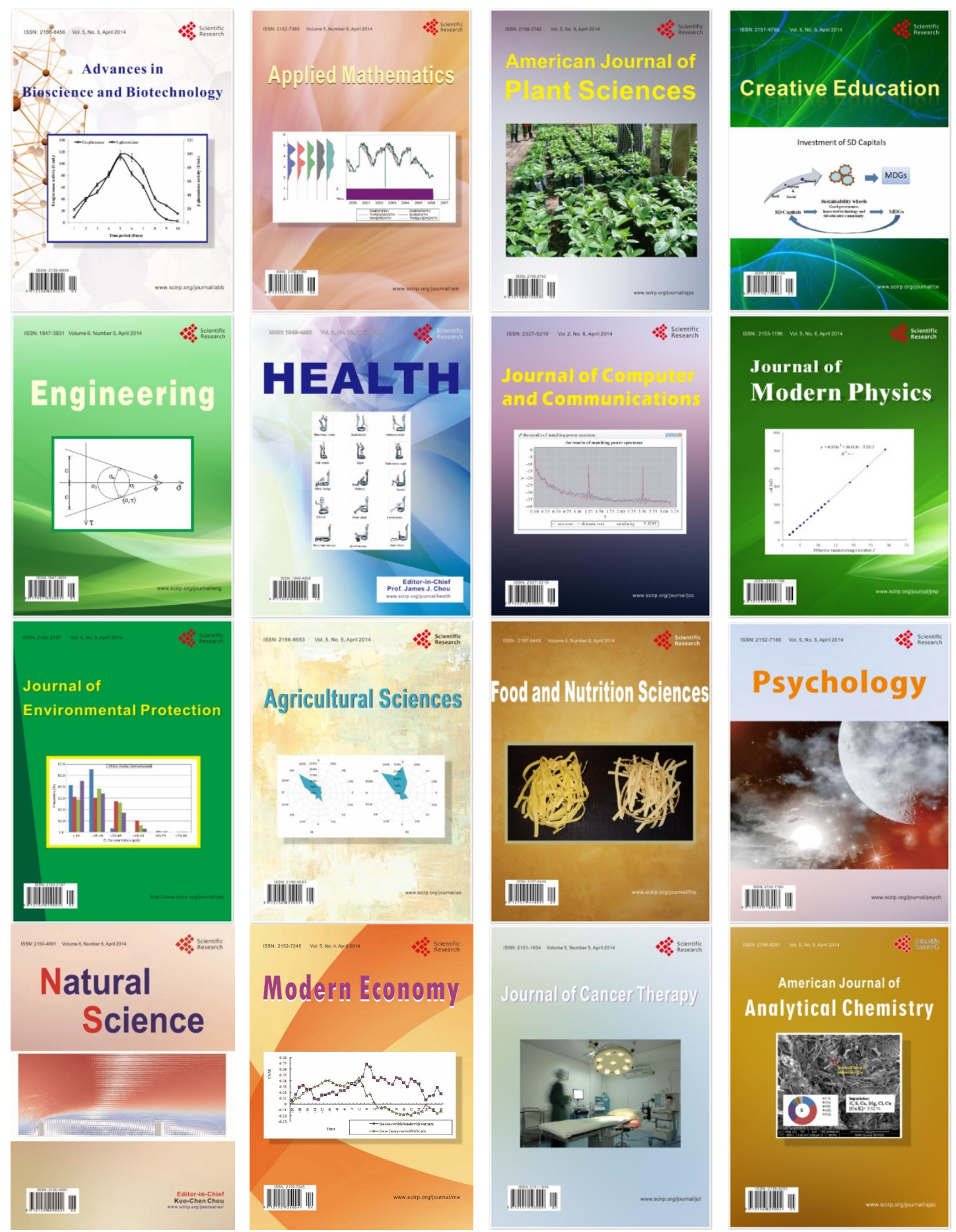